\title{
Effects of Electromagnetic Waves on Brainwaves under Logically Reasoning Status
}

\author{
Fu-Chien Kao ${ }^{1, a}$, Shinping R. Wang ${ }^{1, b}$, Chih-Chia Chen ${ }^{1, \mathrm{c}}$,Yun-Kai Lin ${ }^{1, \mathrm{~d}}$, \\ Chih-Hsun Huang ${ }^{1, \mathrm{e}}$
}

${ }^{1}$ Dept. of Computer Science \& Information Engineering, Da-Yeh University, Taiwan

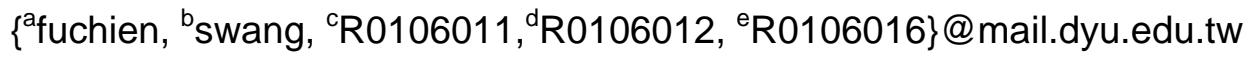

Keywords: EEG; Electromagnetic waves (EMW); WiFi; Brainwave; Reasoning

\begin{abstract}
Nowadays, WiFi becomes a popular wireless accessory method to most of us. People use $\mathrm{WiFi}$ to interact with the wireless Internet, perform commercial and financial transactions, or conducting recreational activities, etc. Though it offers a more convenient life to people, the strong Electromagnetic waves (EMW) resulted from it endangers human health that has already turned out to be the primary study for medical science. The alteration in the sleep EEG in rats due to chronic exposure to low-level non-thermal electromagnetic radiation was investigated. Furthermore, EMW also attracts concern and panic of the inhabitants living in the surroundings which is filled with high-frequency and low-frequency EMW. This paper proposed a design of EEG sensor to detect the logically reasoning brainwaves under electromagnetic wave interference. This study mainly focuses on how EMW produced from WiFi affects subject's brainwaves change under logically reasoning status when human beings expose in various intensity of EMW.
\end{abstract}

\section{Introduction}

Electromagnetic waves are produced by the motion of electrically charged particles. Sometimes, these waves are also called electromagnetic radiation. There are three types of man-made electromagnetic waves surround us and affect our health. They are Radio Waves (RF, also known as Radio Frequency), Extremely Low Frequency (ELF) electric field, and Electric Magnetic Field (EMF) magnetic field. The electromagnetic wave from the cellular phone is RF, and the $50 / 60 \mathrm{~Hz}$ electromagnetic wave from the household electricity falls into the categories of ELF and EMF. Wi$\mathrm{Fi}$, also spelled Wifi or WiFi, is a local area wireless technology that allows an electronic device to exchange data or connect to the internet using $2.4 \mathrm{GHz} \mathrm{UHF}$ and $5 \mathrm{GHz}$ SHF radio waves. WiFi is operating using the high frequencies to transmit and receive signals and it is a commonly known term to describe a method by which various electronic devices, such as personal computers, laptops, tablets and smart phones, video game consoles and some digital audio players, such as the iPod Touch, can wirelessly connect to the Internet. This is done through a localized access point, such as a wireless router in your home, or what is commonly known as a hotspot in a public area, such as a coffee shop, hotel lobby or airport lounge [1].

Therapeutic applications such as soft tissue healing appliances, hyperthermia for cancer treatment, or diathermy expose the patient well above the recommended limit values to achieve the intended biological effects [2]. Burr and Northrop examined and published the effects of stable voltage gradients on various biological systems. They were followed by a lot of scientists who found that stable voltage gradients led to many drastic changes in the organism, including growth and local injury. Studies have shown that these effects were associated with changes in distribution of ions [3]. According to some authors, there is connection with electromagnetic fields and disappearance of bees known as colony collapse disorder in Europe and the US, and that it could also interfere with bird migration [4]. Electromagnetic waves generated by many natural and human-made sources can travel for long distances and play a very important role in daily life. In particular, the electromagnetic fields in the Radiofrequency (RF) zone are used in communications, radio and television broadcasting, cellular networks and indoor wireless systems. Resulting from the technological innovations, the use of electromagnetic fields gradually increases and thus people are 
exposed to electromagnetic waves at levels much higher than those present in the nature [5]. Along with the widespread use of technological products in daily life, the biological effects of electromagnetic waves started to be discussed. Particularly, the dramatically increasing number of WiFi users, rising significant concerns due to its potential damage on people exposed by radio frequency waves [6].

This research from the perspective of cognitive neuroscience investigates the effects of EMW from Wireless Wi-Fi Router to the energy distribution of logically reasoning brainwave characteristic band of human being.

\section{EMW Specifications and Measurement}

A wide frequency range of radio wave $(3 \mathrm{kHz}-300 \mathrm{GHz})$ and microwave scattering in our living environment affect our daily life. Most of these EMW are man-made byproducts of broadcasting, radar communications, satellite communications, medical and industrial manufacturing. More specifically, EMW sources form household microwave oven, cellular phone (including base station), radio broadcasting, computer terminal equipment, medical rehabilitation devices, plastic cutting and molding high frequency industrial machinery, etc. EMW is ubiquitous to our daily life. As the result, the effect of EMW to human health has becoming a concern to the public.

The current permissible EMW exposure regulations for different working environment in Taiwan are given in Table 1 [7]. In general, electric magnetic fields distribute over a wide range of frequencies. For non-ionizing radiation, the frequency starts from extreme low frequency $(0 \mathrm{~Hz})$ to nanometer wave $(300 \mathrm{~Hz})$ are all in the category of EMW. There are controllable and uncontrollable EMW surrounding. Example of controllable EMW surrounding includes laboratory where strength of EMW can be measured and adjust by professionals for protection.

This research adopts TES-92 high-frequency electromagnetic meter to measure the strength of EMW. TES-92 monitors high-frequency radiation over the frequency range of $50 \mathrm{MHz}$ to $3.5 \mathrm{GHz}$ and equips a high sensitivity 3-axis non-directional sensor which make it capable of measuring not only electric fields but also strength of electro-magnetic fields of cross electromagnetic chamber (TEM cells) and electromagnetic anechoic chamber (Absorber rooms). It supports different measurement units and operating modes, and complies most known regulations. The exposure to the high frequency EMW should be minimum for healthy consideration. Especially the high frequency has a greater adverse effect on human health that compares to the low frequency counterpart.

Table 1. The suggested susceptible non-ionizing radiation exposure value of environment suggested by Taiwan ICNIRP

\begin{tabular}{|c|c|c|c|c|}
\hline $\begin{array}{c}\text { Frequency } \\
\text { Range }\end{array}$ & $\begin{array}{c}\text { Electric field } \\
\text { strength } \\
\text { E(V/m) }\end{array}$ & $\begin{array}{c}\text { Magnetic field } \\
\text { strength } \\
\mathbf{H}(\mathbf{A} / \mathbf{m})\end{array}$ & $\begin{array}{c}\text { Magnetic flux } \\
\text { density } \\
\text { B(uT) }\end{array}$ & $\begin{array}{c}\text { Power density } \\
\text { Seq(W/m2) }\end{array}$ \\
\hline$<\mathbf{1 H z}$ & - & $3.2 \times 104$ & $4 \times 104$ & - \\
\hline $\mathbf{1 - 8 H z}$ & 10,000 & $3.2 \times 104 / \mathrm{f} 2$ & $4 \times 104 / \mathrm{f} 2$ & - \\
\hline $\mathbf{8 - 2 5 H z}$ & 10,000 & $4,000 / \mathrm{f}$ & $5,000 / \mathrm{f}$ & - \\
\hline $\mathbf{0 . 0 2 5 - 0 . 8 K H z}$ & $250 / \mathrm{f}$ & $4 / \mathrm{f}$ & $5 / \mathrm{f}$ & - \\
\hline $\mathbf{0 . 8 - 3 K H z}$ & $250 / \mathrm{f}$ & 5 & 6.25 & - \\
\hline $\mathbf{3 - 1 5 0 K H z}$ & 87 & 5 & 6.25 & - \\
\hline $\mathbf{0 . 1 5 - 1 M H z}$ & 87 & $0.73 / \mathrm{f}$ & $0.92 / \mathrm{f}$ & 2 \\
\hline $\mathbf{1 - 1 0 M H z}$ & $87 / \mathrm{f} 0.5$ & $0.73 / \mathrm{f}$ & $0.92 / \mathrm{f}$ & $\mathrm{f} / 200$ \\
\hline $\mathbf{1 0 - 4 0 0 M H z}$ & 28 & 0.073 & 0.092 & 10 \\
\hline $\mathbf{4 0 0 - 2 0 0 0 M H z}$ & $1.375 f 0.5$ & $0.0037 \mathrm{f} 0.5$ & $0.0046 f 0.5$ & - \\
\hline $\mathbf{2 - 3 0 0 G H Z}$ & 61 & 0.16 & 0.20 & \\
\hline
\end{tabular}




\section{Proposed EEG Sensor}

The brainwave is defined as arrhythmic of electric potential between brain cells called neurons and proficiently captured by EEG sensor. Brainwave signals are grouped into four types which are Alpha, Beta, Theta, and Delta. The frequency of alpha wave is from 8 to $12 \mathrm{~Hz}$ and significantly presents when the person is in a relaxed condition or reflecting with closed eyes. Slightly higher from alpha, beta wave's frequency, ranges from 12 to $30 \mathrm{~Hz}$. Delta wave is the lowest frequency range starting from almost zero and can be only up to $4 \mathrm{~Hz}$. It is higher during sleeping mode, whereas Theta ranges from 4 to $7 \mathrm{~Hz}$. The proposed relevant brainwave EEG functional block diagram and sensor module is shown as in Fig.1 and Fig.2 [8]. The brainwave sensor proposed in this research is not only small in size, convenient to carry and easy to operate but also is low in price, and is applicable to being used in various industries in the future compared to the medical grade electroencephalograph. Initially, samples will undergo the interview sessions to answer questioners related to the usage of wireless WiFi. The interview session is normally conducted between five to ten minutes before experimental process. The EEG recording duration was five minutes for logically reasoning stage, giving a total of approximately 25 minutes for 5 samples.

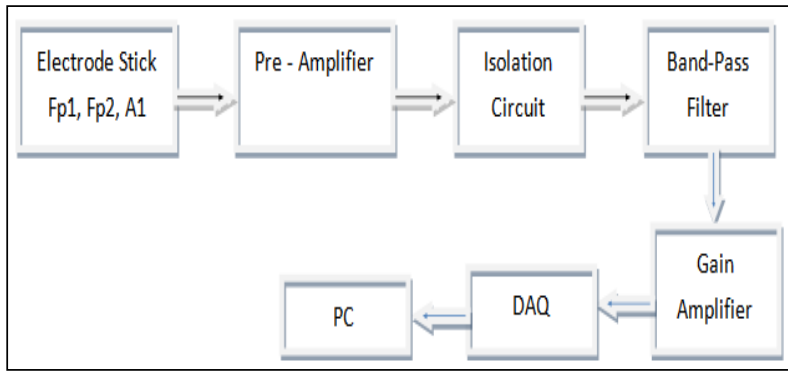

Fig.1. EEG functional block diagram

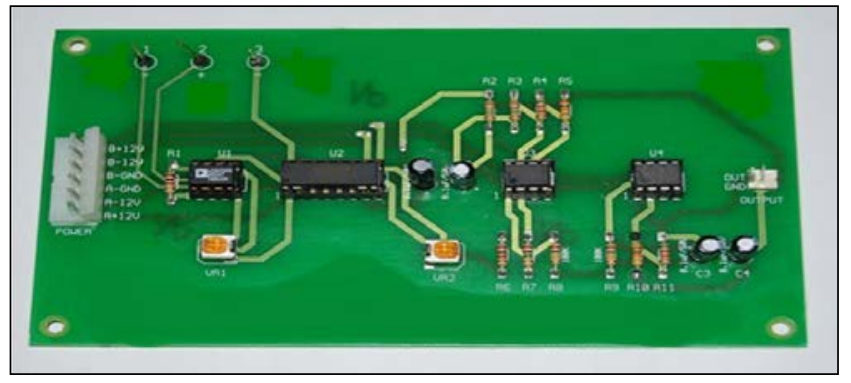

Fig.2. EEG sensor

\section{Proposed Framework of Brainwave Analysis System}

This research analyses the characteristic frequency bands of logically reasoning brainwave from the point of view of the cognitive neuroscience. This research from the perspective of cognitive neuroscience investigates how Electromagnetic waves can influence users' brainwave distribution of characteristic frequency bands. The research experiment accomplishes this by extracting subjects' logically reasoning brainwave under different EMW intensity. The extracted measurements are then analyzed and compiled statistics for its distribution over the logically reasoning brainwave characteristic frequency bands with respect to Electromagnetic waves intensity. The system structure proposed in this research includes the brainwave sensor which is used to capture logically reasoning brainwave signals and the brainwave analysis interface in the back-end which is used to analyze the brainwave frequency bands. The proposed framework of logically reasoning brainwaves analysis is shown in Fig. 3. The related steps are described as follows:

(1). Attach electrode patches to the subjects after installation of a brainwave sensor and capture brainwave signals under logically reasoning status.

(2). Digital brainwave signals are sent to PC and saved as Excel or Txt format through the USB port after being converted by A/D Converter.

(3). Logically reasoning brainwaves analysis interface provides brainwave analysis for the data in the prescribed format. The logically reasoning brainwave signals under different EMW intensity are then processed by formally, as shown in Fig. 4. In this study, the percentages of amplitudes of sectional brainwave frequency band are used to calculate the sectional brainwave energy.

(4).Statistics and analysis of the corresponding characteristic frequency bands energy under different electromagnetic waves intensity.

(5). Compare the difference of logically reasoning brainwave characteristic bands under different electromagnetic waves intensity. 


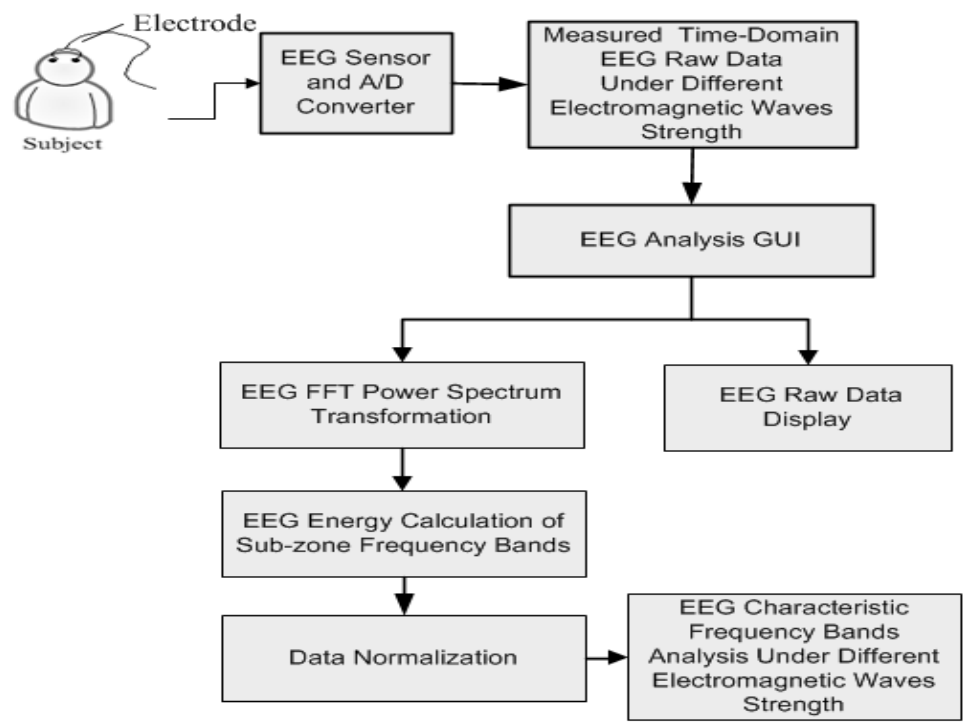

Fig.3. EEG analysis structure under different electromagnetic waves intensity

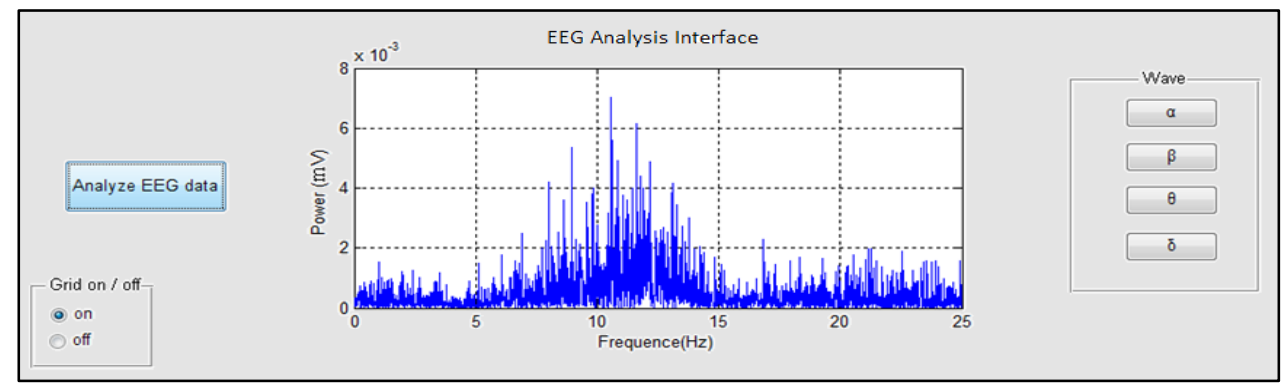

Fig.4. Brainwave analysis interface

\section{Statistics Analysis of Brainwaves Energy under Different WiFi EMW Strength}

The averaged values of the total amplitude of different frequency bands for 5 normal subjects are calculated so as to obtain the energy of the sectional frequency bands and the total energy using Eq. (1) and (2). In the above equations, B is the sectional frequency bands, $f$ is the start frequency of each frequency band, $n$ is the end frequency of each frequency band, and $E$ is energy of each frequency band. $E_{T}$ is the total energy of the four sectional frequency bands from $0.2 \mathrm{~Hz}$ to $25 \mathrm{~Hz}$. The brainwave energy percentage of $\alpha, \beta, \theta$ and $\delta$ is respectively $\left(\mathrm{E}_{\mathrm{B}} / \mathrm{E}_{\mathrm{T}}\right) \%$. The energy percentage of the sub-sectional frequency $E_{\Delta}$ is namely the percentage of the energy in the individual subsection and the energy in the total frequency band, as shown in Eq. (3) [9].

$$
\begin{aligned}
& E_{B}=\sum_{f}^{n} \text { Power }_{f} \\
& E_{T}=\sum_{f=0.2}^{25} \text { Power }_{f} \\
& E_{\Delta}(\%)=\frac{E_{\Delta}}{E_{T}}
\end{aligned}
$$

The averaged brainwaves energy distribution of frequency bands under different EMW strength of $\mathrm{WiFi}$ are listed in Table 2. Experimental results show that $\beta$ brainwaves energy gradually increase and the $\delta$ and $\theta$ waves gradually decrease while the intensity of electromagnetic wave gradually increases. 
Table 2. Averaged logically reasoning Brainwave Energy distribution under different EMW intensity

\begin{tabular}{|c|c|c|c|c|}
\hline \multirow{2}{*}{ EMW strength } & \multicolumn{4}{|c|}{ Brainwaves Energy under logically reasoning } \\
\cline { 2 - 5 } & $\delta \mathrm{Wave}(\%)$ & $\theta \mathrm{Wave}(\%)$ & $\alpha \mathrm{Wave}(\%)$ & $\beta$ Wave $(\%)$ \\
\hline $5-10 \mathrm{uW} / \mathrm{cm}^{2}$ & 15.04 & 16.61 & 20.96 & 47.39 \\
\hline $10-15 \mathrm{uW} / \mathrm{cm}^{2}$ & 14.76 & 15.05 & 21.68 & 48.51 \\
\hline $15-20 \mathrm{uW} / \mathrm{cm}^{2}$ & 10.31 & 13.28 & 21.75 & 54.66 \\
\hline $20-25 \mathrm{uW} / \mathrm{cm}^{2}$ & 9.44 & 10.89 & 21.75 & 57.92 \\
\hline
\end{tabular}

\section{Conclusions}

This research from the prospective of Cognitive Neuroscience investigates the effect of high frequency EMW to the energy distribution of human brainwave characteristic band. The experiment extracts energy of brainwave characteristic band of Wi-Fi users and analyze the changes according to different EWM intensity. The experimental results show that $\beta$ brainwaves energy gradually increase and $\delta, \theta$ brainwaves energy gradually decrease while the intensity of electromagnetic wave gradually increases. This means that the intensity of the electromagnetic wave would actually affect people's brainwaves under logically reasoning.

\section{Acknowledgements}

This research is supported by the grant NSC 102-2511-S-212-001-MY2 from the National Science Council of Taiwan.

\section{References}

[1] C. Sage, D. O. Carpenter: Public Health Implications of Wireless Technologies. Journal of Pathophysiology, Vol. 16(2-3), pp 233-246, 2009.

[2] Possible effects of Electromagnetic Fields (EMF) on Human Health, Scientific Committee On Emerging And Newly Identified Health Risks (SCENIHR), 2010.

[3] H.S. Burr, F.S.C. Northrop: The electro-dynamic theory of life. The quarterly Review of Biology 10(3), 322-333, 1935.

[4] V. P. Sharma, R. N.Kumar: Changes in honeybee behavior and Biology under the influence of cellphone radiations, Current Science, Vol. 98, No. 10, 2010.

[5] P. Vecchia, R. Matthes, G. Ziegelberger, R. Saunders, A. Swerdlow: Exposure to high frequency electromagnetic fields, biological effects and health consequences $(100 \mathrm{kHz}-300$ $\mathrm{GHz}$ ).Review of the scientific evidence on dosimetry, biological effects, epidemiological observations, and health consequences concerning exposure to high frequency electromagnetic fields. International Commission on Non-Ionizing Radiation Protection, ICNIRP, 2009.

[6] M. Roosli, E. Mohler and K. Hug: 'Systematic review on the health effects of exposure to radiofrequency electromagnetic fields from mobile phone base stations', Bull World health Organ:88:887-896. www.intechopen, 2010.

[7] Non-ionizing Radiation Regulations in Taiwan, Bureau of Health Promotion in Taiwan

[8] F. C. Kao, H.C. Hsieh and W. T. Li: ‘Analysis of Brainwave Characteristic Frequency Bands for Learning’, 11th IEEE International Conference on Bioinformatics \& Bioengineering, pp.314317, 2011.

[9] F.C. Kao, J. H. Jhong :Analysis of Brainwave Characteristic Frequency Bands for Logic Reasoning. Applied Mechanics and Materials.Vol.145, pp.470-474, 2012. 